\title{
The diagnosis and treatment of pulmonary valve stenosis in children
}

\author{
Shiv Kumar Yadav \\ Department of Pediatrics, Shanghai United Family Hospital and Clinics, Shanghai, China
}

\section{A B S T R A C T}

Pulmonary valve stenosis (PS) is a common congenital heart disease, its early diagnosis and treatment can improve growth and prognosis in children with pulmonary valve stenosis. With the development of the diagnostics and treatment, percutaneous balloon pulmonary valvuloplasty (PBPV) has replaced surgery as the first choice for isolated pulmonary valve stenosis. The article reviews the recent progress in diagnosis and treatment of pulmonary valve stenosis.

Key words: Percutaneous balloon pulmonary valvuloplasty, Pulmonary valve stenosis, Surgery, Children

\section{INTRODUCTION}

Congenital heart disease (CHD) is the embryonic variety of internal and external factors affecting cardiovascular development. According to statistics in the range of the incidence of live births is $4 \%$ to $50 \%$; ${ }^{1}$ which the pulmonary valve stenosis (PS), about $5.1 \% .^{2}$

\section{Types of pulmonary stenosis}

According to the pathological anatomy of the PS divided into 3 types:

(1) Valve type: is the most common, and Milo, the type is further divided into three subtypes: dome type $(60 \% \sim 70 \%)$, dysplasia type \& hourglass-like deformity with bottle-like sinus node type. ${ }^{3}$

(2) Inferior Valve (RV outlet stenosis)

(3) Superior Valve

Pulmonary stenosis severity based on the right ventricle and pulmonary artery pressure difference may be divided into mild, moderate and severe. Right ventricular pressure and pulmonary transvalvular pressure gradient $(\Delta \mathrm{P})$ is greater than $10 \mathrm{~mm} \mathrm{Hg}(1 \mathrm{~mm} \mathrm{Hg}=0.133 \mathrm{kPa})$, can be diagnostic for pulmonary valve stenosis; a quiet state, the right ventricular systolic pressure $>30 \sim 50 \mathrm{~mm} \mathrm{Hg}$, and $\Delta \mathrm{P}>10 \sim 40 \mathrm{~mm} \mathrm{Hg}$ for mild pulmonary valve stenosis; right ventricular systolic pressure greater than $50 \mathrm{mmHg}$, but less than left ventricular systolic pressure, $\Delta \mathrm{P}>41$ $\sim 100 \mathrm{mmHg}$ for moderate pulmonary stenosis; right ventricular systolic pressure greater than left ventricular systolic pressure, $\Delta \mathrm{P}>100 \mathrm{mmHg}$ for severe pulmonary valve stenosis.

\section{Diagnosis}

\section{Clinical Features}

Pulmonary Valve Stenosis clinical symptoms and signs are not obvious for mild and moderate type but if sometimes patients do some exercise, they may have shortness of breath and etc; some children have cylindrical month-like face. In typical cases, systolic thrill can be felt in the left sternal $2^{\text {nd }}$ intercostal-space at the same time heard a rough and loud ejection systolic murmur, murmur often heard on left neck and back side transmission. Some patients may have early systolic clicks sound. The mechanism may suddenly open the thick, fixed a narrow pulmonary valve. P2 significantly reduced or cannot be heard; sometimes you can hear the P2 split, because of the delay extension of the right ventricular ejection time and pulmonary valve. If right ventricular enlargement and tricuspid regurgitation, we may hear full-systolic murmur at the left sternal border in the fourth intercostal. In the most severe PS children, due to right ventricular, right atrial pressure increased so 
that we can find the opening up of atrial septal foramen ovale, atrial right to left shunt, appear peripheral cyanosis in cry, irritable even quiet state; In addition, it also can appear heart failure.

\section{Echocardiography}

$\mathrm{M}$ mode ultrasound visible right ventricular or septal hypertrophy, pulmonary valve thickening, limited opening, \& dilation of pulmonary artery. While Doppler ultrasonography in the diagnosis of PS has a special value, can provide more information such as right ventricular, pulmonary artery pressure and the systolic pressure gradient across the pulmonary valve, typical information of the typical flow spectrum and blood flow velocity. PS can be diagnosed on the basis of Doppler echocardiography, figure out the orifice constriction's diameter \& area and judge its severity.

\section{Electrocardiography (ECG)}

PS presents in children due to right ventricular systolic overload \& the main performance of right ventricular hypertrophy. Therefore, the ECG may appear right axis deviation; increased right heart leads R-wave voltage, such as: $\mathrm{qR}, \mathrm{R}, \mathrm{rR}$ or Rs type; ST-segment depression, $\mathrm{T}$ wave inversion; $\mathrm{S}$ wave deepen to left ventricular lead, while T-wave up-right, are the significant finding for the performance of severe right ventricular hypertrophy. Meanwhile, we can check $V_{1} R$ wave amplitude to estimate right ventricular pressure, transvalvular pressure then to determine the degree of PS. Moreover, The $V_{1} R$ amplitude plays a supporting role on postoperative follow-up visit.

\section{Chest X-Ray}

In mild \& moderate PS, heart size is normal but in the severe PS, such as cardiac function is acceptable with cardiac compensatory mild enlarged. Consider there is a heart failure; the heart is enlarged significantly, mainly for the expansion of the right ventricle and right atrium. About $90 \%$ of the children with PS can be seen the widening of the main pulmonary artery, but in infancy, expansion is not prominent.

\section{Computerized Tomography (CT)-Scan}

At present CT examination, especially in multi-slice CT (MSCT) as a non-invasive technique, can accurately diagnose CHD with or no abnormalities and malformations of the vascular structures, is a reliable way for CHD diagnosis. ${ }^{4}$ With the persistence of the narrow naked valvular ring, the expansion of the artery and hypertrophy of the right ventricle, MSCT can indirectly diagnose PS. However, MSCT image quality is often affected by heart rate and breathing movement. Now we often use ECG-gated scanning mode to avoid the impact. ${ }^{5}$ In this mode, MSCT can be used for the evaluation of cardiac function, such as wall motion, ventricular ejection fraction and cardiac valvular motion, but can't provide like as valve reverse flow and physical aspects such as cardiovascular cavity pressure curve, blood oxygen saturation, and so on, ${ }^{6}$ it requires a combination of data of echocardiography. In addition, there are reported that 64-slice CT angiography for the diagnosis of PS assisted Cardiovascular Angiography. ${ }^{7}$

\section{Magnetic Resonance Imaging (MRI)}

The early 1980 s MRI started to use for diagnosis of the CHD, but early application range limited to provide anatomical information. ${ }^{8}$ The research of Shaolin LI et al. ${ }^{9}$ proved that the sensitive rate of MRI is $96.88 \%$, the specific rate is $93.33 \%$, and the total diagnosis is $93.18 \%$. For the CHD with great vessels abnormalities, especially complex CHD, MRI is quite good to show the adjacent relationship to the heart and helping the diagnosis. Therefore, the MRI should be routinely done for complex $\mathrm{CHD}^{10}$ In addition, there are also reported that enhanced 3-D magnetic resonance imaging is used for angiography. ${ }^{11}$

\section{Cardiac Catheterization and Cardiovascular Angiography}

Cardiac catheterization is the gold standard for diagnosis of PS and determines the extent, ${ }^{12-13}$ the right ventricle pressure, pulmonary artery pressure and systolic pressure gradient across the pulmonary valve can be measured. In cardiovascular angiography PS often presents with dome and spurt sign.

\section{Treatment}

\section{Surgical Treatment}

As early as in 1913, Deyn has tried through the ventricle with a flat knife to cut a narrow valve but didn't get success. Since then, Sellors and Brock successfully did that by the right ventricle. After a difficult exploration, Varco in 1951 first succeeded in blocking the circulation downstream pulmonary valve look directly at the junction of the incision. Swan and others went a step further in 1953; they have taken by pulmonary valvotomy surgery under hypothermic circulatory arrest. In 1958, McGoon and Kirklin have been using hypothermic cardiopulmonary bypass technology purposes to look into the pulmonary valve incision to be successful and it becomes standardization scheme for PS surgical treatment.

The indications of surgical treatment

(1) the systolic pressure gradient across the pulmonary valve $>40 \mathrm{mmHg}$, with or without clinical symptoms; (2) ECG suggests right ventricular hypertrophy, myocardial strain; (3) PS with other heart malformations required surgical treatment; (4) Severe PS with right ventricular outflow tract obstruction for the pulmonary valve incision plasty, and concomitant right ventricular outflow tract patch. (5) For small pulmonary valve ring, the valve leaflet thickening and stiffness of the pulmonary valve dysplasia as well as with 
right ventricular outflow tract stenosis in children, catheter interventional treatment is ineffective, ${ }^{14}$ (6) For some right heart function low with severe PS children, where catheter interventional treatment has higher risk. (7) For children; PBPV treatment or treatment failure (8) involved in the treatment of interventional therapy at the same period (hybrid surgery).

\section{Age for surgery}

The ideal age for surgery is 5 to 12 years old but should not be commonly $>25$ years. In addition, symptoms of right heart failure of any age can be considered surgery. The systolic pressure gradient across the pulmonary valve $>100 \mathrm{mmHg}$ in children with PS (i.e., severe PS), has to do operation as soon as possible.

\section{Surgical methods}

(1) Hypothermic circulatory occlusion under direct vision pulmonary valve incision: As the time limit for the hypothermic circulatory arrest, applies only to the pure PS cases. (2) Cardiopulmonary bypass pulmonary valve look directly at incision: Compared with the former, this mode can provide sufficient time to identify lesions. It can be divided into extracorporeal circulation heart valve incision under herein \& cardiac arrest under cardiopulmonary bypass valve incision, the former is applicable to pure PS, and the latter in other valve stenosis or in combination with other serious intra-extra cardiac abnormalities cases.

The surgical mortality reported in the literature of PS of 0 to $20 \% .{ }^{15}$ U.S. Department of Thoracic Surgery Statistics PS operative mortality was $2.5 \%$, European literature reported case fatality rate of $7 \%{ }^{12}$ In $5 \%$ to $10 \%$ cases postoperatively, re-stenosis occurs and which need second surgical treatment. For pulmonary hypoplasia and critical neonatal PS, restenosis rate is even higher; it is often accompanied by varying degrees of pulmonary regurgitation post-operatively. The mortality rate of China's Beijing Fuwai Hospital was 2.3\% ${ }^{15}$

Surgery common complications: Damage of pulmonary arterial wall, High-pressure residual right ventricle and pulmonary valve regurgitation, even the hypoxemia, acute lung injury and right ventricular dysfunction. Respiratory complications and hypoxia are the major cause of death. ${ }^{15}$

\section{Interventional Therapy}

In 1982, Kan \& others ${ }^{16}$ reported percutaneous balloon pulmonary valvuloplasty (PBPV), thereafter the application of catheter interventional treatment for PS rapidly developed. At present, the most PS can be cured through PBPV and only a few are not suitable before considering surgical treatment.
The clear indications of PBPV

a) Typical PS, b) Normal cardiac output and the systolic pressure gradient across the pulmonary valve, checked by the cardiac catheterization $\geq 40 \mathrm{mmHg}$, c) $2 \sim 4$ years old is the best age and the remainder all ages can be carried out. ${ }^{17}$

Relative indications

(1) Typical PS, ECG showed right ventricular and right ventricular angiography showed pulmonary expansion, jet flow existence but the cardiac catheterization inspection $35 \mathrm{mmHg} \leq$ across the pulmonary valve pressure $<50 \mathrm{mmHg}$; (2) neonates with severe PS; (3) severe PS with atrial level right to left shunt; (4) mild to moderate dysplasia type PS; (5) the typical PS with patent ductus arteriosus or atrial septal defect and so on, can be simultaneously involved in interventional treatment. ${ }^{17}$ The relative indications are choosing to be based on each cardiac catheterization experience, conditions, personnel and equipment and children circumstances for each cardiovascular interventional center.

Non-appropriate indications

(1) Inferior valve type with a normal valve;

(2) severe dysplasia type PS;

(3) with severe tricuspid regurgitation required surgical treatment. ${ }^{17}$

\section{PBPV Operation Method}

Conventional in general anesthesia or under local anesthesia, intubation canula in femoral vein, and before the balloon catheter is inserted and the expansion needs to expanding and sucking deflated balloon several times with the dilute contrast agent, in order to check for damage or breakage balloon, and remove the air. ${ }^{17}$ According to illness condition, we can choose of single, double or triple balloon angioplasty.

Single Balloon Method

Puncture the femoral vein in the groin, the floating catheter via the femoral vein, inferior vena cava, right atrium, pulmonary artery, usually to the left pulmonary artery, and finally reach the small pulmonary arteries, continuous curve measured of pulmonary artery to right ventricular pressure difference, parallel, right ventricular angiography in order to determine the pulmonary artery prosthetic ring diameter. Commonly used balloon diameter to pulmonary valve ring diameter is of 1.2 1.3 times. Then insert the guide wire to the left lower lobe artery; along the wire balloon catheter is sent to the pulmonary valve, the central of balloon fixed on then expand the balloon 3 to 4 times, each time filling time $4 \sim 5$ s. If the expansion success, generally visible balloon "waist" sign suddenly disappears, on physical examination, pulmonary valve area tremor is relieved or disappeared, and cardiac murmur is significantly reduced. After balloon 
dilation, repeat right heart catheterization to record pulmonary artery to right ventricular continuous pressure curve, measure the systolic pressure gradient across the pulmonary valve, and right ventricular angiography observe the effect of balloon dilatation.

\section{Double Balloon Method}

In order to achieve adequate balloon annulus ratio, some cases require for the double-balloon dilatation. The sum of the two commonly used balloon diameter to pulmonary valve ring diameter of 1.5 times or slightly more. Choose two roughly the same diameter and length of the balloons, puncture from the left and right femoral vein to insert a balloon catheter, with the single balloon technique.

Double \& Three Balloon Method

Operate with a single balloon method. Puncture the femoral vein and send with the super-hard guide-wire. At first expand just one balloon 1 or 2 times and then the others two or all at the same time. Monitor balloon inflation pressure, and observe the balloon waist concave. The appropriate balloon inflation pressure is about to $2.0 \sim 8.5 \mathrm{~atm}$.

\section{PBPV Balloon Selection}

PBPV balloon have Inoue balloon, Mansfield balloon, Baxter balloon, double leaf and trefoil balloon, etc. Three leaves balloon consisted of 3 same size $4 \mathrm{~cm}$ long balloons around the same catheter; can synchronize filling expansion and have part of blood flow in the gap of balloon. At present, Inoue balloon is the most widely used. Generally cases are treated with a single balloon dilatation, for the greater valve, can use double-balloon angioplasty technology. In addition, because of the unique advantages of the trefoil balloon, has been adopted in clinical practice gradually. ${ }^{18}$

The key of PBPV to success is the balloon/annulus diameter ratio. For simple PS, Balloon/annulus diameter ratio of 1.2 to 1.4 , is more appropriate. For relative indications of mild to moderate dysplasia PS often choose larger balloon/annulus ratio (1.4 to 1.5), in order to get satisfied results. While for the severe PS, often use a smaller balloon for expansion first and then use larger balloon but the expansion of the number should not be too much, otherwise, there may be a reactive infundibular spasm.

Most children's right ventricle - pulmonary artery systolic pressure can be reduced to the normal range postoperatively, but the indications to improper selection, the effect is often not ideal. For postoperatively re-stenosis cases, can try again for PBPV operation.

\section{Complications of PBPV Operation}

The complications reported in the literature, about $4.5 \%$, of which about $0.6 \%$ were major complications, including: death by $0.2 \%$, heart perforation to cardiac tamponade $0.1 \%$ and tricuspid regurgitation $0.2 \%$; Minor complications and other $3.9 \% ;^{19}$ also can appear hypotension, arrhythmia, tricuspid valve chordae loss. ${ }^{17}$

Long-term follow-up showed that; 6 months of postoperative, the systolic pressure gradient across the pulmonary valve stabilized; ${ }^{20}$ right ventricular hypertrophy and right ventricular enlargement gradually reduced; Generally PBPV postoperative 6 to 12 months, right ventricular diastolic function returns to normal, ${ }^{19}$ long-term effectiveness was same in comparison with surgical operation. ${ }^{21}$

Overall, the hospital stay, postoperative ICU monitoring time, the average operation time-consuming and postoperative complications and mortality, etc of PBPV are significantly lower than the surgery. At present, it is basic alternative to surgical operation and has become the preferred method of treatment of pure PS. ${ }^{22-23}$ Definitely, unsuitable cases for PBPV and serious complications after PBPV all need to do open surgery to compensate.

\section{AKNOWLDGEMENT}

None

\section{REFERENCES}

1. Hoffman JI, Kaplan S. The incidence of congenital heart disease. J Am Coll Cardiol 2002;39 (12): 1890-1900.

2. Yang Jiang-fan, Xu Yue-Zhen, Li ping. 20436 Infants with congenital heart disease epidemiology investigation in Jiaxing city. The Journal of Pediatrics 1997; 35 (8): 417-420.

3. Rao PS. Transcatheter treatment of pulmonary outflow tract obstruction: A review. Prog Cardiovasc Dis 1992, 35(2):119-158.

4. Qian Juan, Wang Ying, Yan Yong. Fiberoptic bronchoscopy and multidetector -row CT in children with congenital heart disease airway lesions in the diagnosis of application is more. Chinese Pediatric Emergency Medicine 2007; (6): 508-510.

5. Goo HW, Park IS, Ko JK, Dong-Man Seo, Jeong-Jun Park. Computed tomography for the diagnosis of congenital heart disease in pediatric and adult patients. Int $\mathrm{J}$ Cardiovasc Imaging 2005, 21 (2-3):347-365.

6. Matsui H, Yasukochi S, Hasevama K. Quantification of right and left ventricular volumes in children with congenital heart disease by multidetector-row computed tomography. Pediatr Cardiol 2007, 28(4):267-271.

7. Khatri S, Varma SK, Khatri P, Raghavannair SK. 64-Slice muhidetector -row computed tomographic angiography for evaluating congenital heart disease. Pediatr Cardiol 2008; 29(4):755-762.

8. Fellows KE and Hubbard AM. MRI: Complacency or ascendancy in the evaluation of congenital heart disease.Pediatr Radiol 1999;29(7):491-495.

9. Li Shao-lin, Huang Ji-Liu and Li Yizhuo. Congenital heart disease MRI (magnetic resonance imaging) diagnosis method and clinical application! Chinese Journal of Radiology 1994; 28(7): 482-484. 
10. Hu XiGong and Huang Guo-Ying. Magnetic resonance imaging diagnosis of congenital heart disease research. International Journal of Pediatrics 2007; (6): 428-430.

11. Geva T, Greil GF, Marshall AC, Landzberg M and Powell AJ. Gadolinium-enhanced 3-Dimensional magnetic resonance angiography of pulmonary blood supply in patients with complex pulmonary stenosis or atresia comparison with X-Ray angiography. Circulation 2002; 106 (4):473-478.

12. Roos-Hesselink JW, Meijboom FJ, Spitaels S, vanDomburg RT, vanRijen EHM, Utens EMWJ, et al. Long-term outcome after surgery for pulmonary stenosis (a longitudinal study of 22-33 years). Eur Heart J 2006; 27(4):482-488.

13. Sharieff $S$, Shah-e-Zaman $K$ and Faruqui AM. Short-and intermediate-term follow-up results of percutaneous transluminal balloon valvuloplasty in adolescents and young adults with congenital pulmonary valve stenosis. J invasive Cardiol 2003; 5(9): 484-487.

14. Vancini M, Roberts KD, Silove ED and Singh SP. Surgical treatment of congenital pulmonary stenosis due to dysplastic leaflets and small valve. J Thorac Cardiovac Surg 1980; 79 (3):464-468.

15. Wu Qing-Yu. Heart surgery. Jinan, Shandong science and technology press 2003; 373-376.

16. Kan JS, White RI, Mitchell SE and Gardner TJ. Percutaneous balloon valvuloplasty: A new method for treating congenital pulmonary valve stenosis. N Engl J Med 1982; 307(12):540-542.
17. Zhou Ai-Qing and Jiang Shi-Liang. Congenital heart disease transcatheter therapy guidelines! The Journal of Pediatrics 2004; (3): 234-239.

18. Escalera II RB, Chase TJ and Owada CY. Triple-balloon pulmonary valvuloplasty: An advantageous technique for percutaneous repair of pulmonary valve stenosis in the large pediatric and adult patients. Catheter Cardio Inte 2005; 66 (3):446-451

19. Stanger P, Cassidy SC, Girod DA, Kan JS, Lababidi Z and Shapiro SR. Balloon pulmonary valvuloplasty: Results of the valvuloplasty and angioplasty of congenital anamalies registry. Am J Cardiol 1990; 65 (11):775-783.

20. Marantz PM, Huhta JC, Mullins CE, Murphy DJ, Nihill MR, Ludomirsky A, et al. Results of balloon valvuloplasty in typical and dysplastic pulmonary valve stenosis: Doppler echocardiographic follow-up. J Am Coll Cardiol 1988; 12 (2):476-479.

21. Jarrar M, Betbout F, Farhat MB, Maatouk F, Gamra H, Addad F, et al. Long-term invasive and noninvasive results of percutaneous balloon pulmonary valvuloplasty in children, adolescents, and adults. Am Heart J 1999; 138(5):950-954.

22. Ling Jian, Xie ReLan, Xu Li. Percutaneous balloon angioplasty pulmonary valve experiences and their efficacy analysis of rotational acetabular osteotomy, Chinese Journal of Cardiovascular Disease 2003; 31 (5): 323-325.

23. Rao PS. Percutaneous balloon pulmonary valvuloplasty: State of the art. Catheter Cardio Inte 2007, 69(5):747-763.

Authors Contribution:

SK Yadav: Designed the study, collected the data, analyzed the data, drafted the manuscript \& reviewed the manuscript.

Source of Support: Nil, Conflict of Interest: None declared. 